\title{
Synthesis of high oleic palm oil-based trimethylolpropane esters in a vacuum operated pulsed loop reactor
}

\begin{abstract}
The experimental studies have shown that a pulsed loop reactor can be used to successfully reduce the reaction time for the transesterification of oils and fats. In the present study, the transesterification of palm methyl ester (PME) and trimethylolpropane (TMP) to produce palm oil based TMP ester was conducted using the vacuum operated pulsed loop reactor. The reaction was catalyzed with sodium methoxide solution in methanol. The influences of five operating variables such as vacuum pressure, catalyst loading, molar ratio of high oleic PME to TMP, reaction temperature and oscillatory speed on the yield of TMP esters and unwanted fatty soap formation were examined. The optimum conditions for the reaction were found at $120{ }^{\circ} \mathrm{C}, 20 \mathrm{mbar}, 3.9: 1 \mathrm{M}$ ratio of PME:TMP, $1.0 \mathrm{wt} \%$ catalyst solution and $180 \mathrm{rpm}$ oscillatory speed. The product containing $95 \mathrm{wt} \%$ TMP triester was successfully synthesized in $1 \mathrm{~h}$ with $167 \mathrm{mg} / \mathrm{g}$ of fatty soap. The physicochemical properties of the TMP esters obtained using pulse reactor were comparable to characteristics of high oleic TMP esters.
\end{abstract}

Keyword: Biolubricant; Trimethylolpropane ester; Pulsed loop reactor; Methyl ester 